\title{
Recognition of Offline Handwritten Characters using 2D-FFT for English and Hindi Scripts
}

\author{
Panyam Narahari Sastry, Syed Sameer, Mohammed Sameer Syed
}

\begin{abstract}
The Handwritten Character Recognition has been a challenging task for the past many decades. This is an old application related to the area of pattern recognition. Handwritten character recognition (HCR) can be classified into two types namely, Online and Offline. As per the literature survey, there are no standard databases for HCR ${ }^{[1][2][3]}$ since there are very less number of speakers for any Indian language compared to English. Hence, the database of Indian scripts both for testing and training are to be developed in the laboratory environment. The recognition accuracy for printed / typed characters is more than 99 percent, whereas for the HCR it is around 60 percent. Hence the area of HCR is an open area of research. HCR for Indian languages is at nascent stage compared to English since they contain alphabets and also matra's / sandhi are complex which make the recognition tougher. The freedom of the scriber in writing the script is also another challenge for achieving the better recognition accuracy. This work describes the handwritten character recognition of both Hindi and English scripts by extracting features using $2 D$ FFT and using the Nearest Neighborhood Classifier. The best recognition accuracy for handwritten character recognition of English and Hindi languages obtained is $70 \%$.
\end{abstract}

Keywords : 2D FFT, Handwritten Character Recognition, Nearest Neighborhood Classifier, Pattern Recognition.

\section{INTRODUCTION}

Optical Character Recognition goal is to identify characters in images of printed or handwritten text, in order to encode the text in a format which is more convenient to be edited, copied on papers, or may be distributed electronically across world-wide networks. One of the most popular natural ways of generating paper documents is handwriting. Most paper documents are handwritten because it is a natural means with convenience and speed. Wide spread acceptance of digital computers seemingly challenges the future of handwriting as the keyboard is becoming the most popular means of generating text. But the convenience of paper and pen is the reason that still handwriting persists in the age of digital computer and hence seen as a more natural way to enter text

Revised Manuscript Received on April 18, 2020.

* Correspondence Author

Dr. Panyam Narahari Sastry*, Professor, Department of ECE, Chaitanya Bharathi Institute of Technology (A), Hyderabad, India.

Syed Sameer, PG, Department of ECE, Chaitanya Bharathi Institute of Technology (A), Hyderabad, India.

Mohammed Sameer Syed, PG, Department of ECE, Chaitanya Bharathi Institute of Technology (A), Hyderabad, India.

(C) The Authors. Published by Blue Eyes Intelligence Engineering and Sciences Publication (BEIESP). This is an open access article under the CC BY-NC-ND license (http://creativecommons.org/licenses/by-nc-nd/4.0/) into a computer, provided computers can decode the handwriting. In offline HCR systems, the recognition is done only after all the writing is complete. The input is the raw handwritten data, usually in the image form and involves analysis of the two-dimensional image. The major advantage of the offline systems is to allow previously written texts to be processed and then to be recognized.

To construct more precise results, the following needs to be utilized.

- Minimize the Noise effect. The input image is usually noisy due to a number of factors like writer, paper, pen, ink and scanner quality and has a high influence on the recognition.

- $\quad$ Segment the text. The input text image may be of a page of a document, a word or a character at a time. To convert an image with a page of document to soft form, the lines of text are to be first segmented. From the line, the words are to be segmented. The words can be used as a whole for its recognition or it can be further segmented into characters.

- Handle ambiguity. There is no opportunity for feedback or user interaction in case of ambiguity or dead end as completed writing is available as an image and the recognition happens often in the absence of the writer's involvement.

- Improve the recognition quality. Even 99\% accuracy of HCR system translates to 30 errors on a typical printed page of 3000 characters and correcting such random errors requires enough time and an alert proofreader. Hence for off-line systems, the recognition speed may not be an issue, but the recognition quality, definitely is an issue.

The drawbacks of these systems are:

- Need for robust preprocessing techniques to make the data suitable for recognition.

- The temporal information is absent and hence stroke level processing needs complex steps to extract strokes from the image, but, the sequence in which these strokes are generated cannot be regenerated.

- Segmentation of the characters in case of overlapping, cursive or mixed scripts is difficult.

\section{METHODOLOGY}

In the literature it is evident and very clear that there are no standard databases for Indian languages for the purpose of handwritten character recognition ${ }^{[1] ~[2] ~[3] . ~ F u r t h e r, ~ i t ~ i s ~}$ recommended in the literature to develop handwritten characters in the laboratory environment ${ }^{[1][2][3]}$.

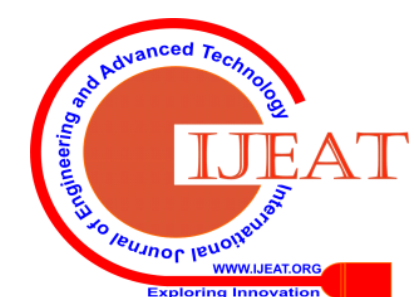


Hence the researchers working in this area develop handwritten characters. This is the first step in handwritten character recognition. The database is used for both training and testing the algorithm. In the second stage the researchers identify different features and combinations of different features to get the maximum recognition rate. After features selection the algorithms are developed to classify and identify the various handwritten characters.

In this project the database represents 15 classes of Hindi characters, 15 classes of English characters and then 15 classes of both Hindi and English characters. Each class consists of 50 samples out of which some are for training and testing. Each character is written on a paper in a rectangular box in different sizes. The handwritten documents were then obtained by scanning them with a scanner. These images are preprocessed individually and using the minimum boundary rectangle concept, normalization is performed to a size of $50 \times 50$.

The following flowchart is the step by step algorithm for 2-D FFT implemented on basic and isolated handwritten characters ${ }^{[6]}$.

\begin{tabular}{|c|}
\hline The images size of $50 \times 50$ pixels are to be loaded \\
\hline Read all the images \\
\hline 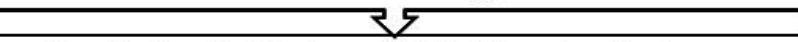 \\
\hline Use the threshold value of 0.7 to binaraize the images \\
\hline Z \\
\hline For all the images find the $2 \mathrm{D}-\mathrm{FFT}$ \\
\hline 2 \\
\hline $\begin{array}{l}\text { Each image to be formed as feature vector by reshaping the } \\
\text { matrix into column matrix }\end{array}$ \\
\hline Z \\
\hline The above procedure is to be repeated for test images too \\
\hline Find out the distance between the column matrix of the test \\
\hline $\begin{array}{l}\text { Find out the distance between the column matrix of the test } \\
\text { image and each training image }\end{array}$ \\
\hline 5 \\
\hline Find out the minimum distance \\
\hline Z \\
\hline $\begin{array}{l}\text { The match count to be incremented if class matches else } \\
\text { increment the mismatch count }\end{array}$ \\
\hline 5 \\
\hline
\end{tabular}

The Mathematical model of 2D-FFT includes a number of one dimensional Fourier transforms. The definition of 2D FFT equation (1) shows the 2D FFT of the image $f(p, q)$ and equation (2) represents inverse 2D FFT. The 2D FFT F $(\mathbf{s}, \mathbf{t})$ for the image $f(p, q)$ can be found using formula

$$
F(s, t)=\frac{1}{M N} \sum_{p=0}^{M} \sum_{q=0}^{N} f(p, q) e^{-j 2 \pi\left(\frac{5 p}{M}+\frac{t g}{N}\right)}
$$

Where $\mathrm{s}=0,1,2 \ldots \mathrm{M}$

$$
\& \mathrm{t}=0,1,2 \ldots . \mathrm{N}
$$

Here $p, q$ are the pixel coordinates in the image and $s$, $t$ are coordinates in the "transformed image". The inverse 2D FFT (from FFT back to the original image, possibly after filtering) can be obtained using formula

$$
f(p, q)=\sum_{s=0}^{M} \sum_{t=0}^{\mathrm{N}} \mathrm{F}(s, \mathrm{t}) \mathrm{e}^{\mathrm{j} 2 \pi\left(\frac{5 \mathrm{~s}}{M}+\frac{\mathrm{tg}}{\mathrm{N}}\right)}
$$

$$
\begin{aligned}
\text { Where } p & =0,1,2 \ldots M \\
\& q & =0,1,2 \ldots . N .
\end{aligned}
$$

these formulae assume calculation using complex numbers

$$
(j=\sqrt{(-1)}) \text {. }
$$

\section{RESULTS AND DISCUSSIONS}

All the experiments are performed over a system having a 64 bit operating system, and Intel (R) Core(TM) i7-8750H CPU@ @ 4.41 GHz and were done through MATLAB R2014b. The images were scanned and saved in the computer. Primarily by using Adobe Photoshop the images are normalized to 50x50 pixels size and then applying a threshold of 0.7. The pixel intensities are binarized. The 2D-FFT is applied to these binary images and the real part of the transform value (of the pixel) is considered for the experimentation. Later, the Euclidean distance is calculated between each test image and database images and the minimum Euclidean distance is considered from the database image to the test image. The output is represented where the test image is on the left side and the matched image of the database is on the right side.

There are 3 database sets prepared in this work. The first data base set consists of Hindi handwritten letters 15 in number (i.e. 15 classes). Each class again has 50 samples. Therefore, total number of handwritten Hindi characters become 750 images. Similarly for the English handwritten letters, a separate database consisting of 15 classes and 750 images were developed in the laboratory. The last database set consists of both Hindi and English letters then we get 1500 images.

The table 1 shows the results obtained for the 15 Hindi classes. The number of training samples and testing samples were varied and different recognition accuracies (R.A) were obtained. The best R.A for the Hindi script obtained was 72\%. The R.A increased as the number of training samples have increased. It was observed that when the number of training samples were 45 and the testing samples were 5, the R.A was best and found to be $72 \%$.

Table- 1: Experiment 1 for 15 Hindi classes

\begin{tabular}{|c|c|c|c|}
\hline S.no. & $\begin{array}{c}\text { No. of training } \\
\text { samples }\end{array}$ & $\begin{array}{c}\text { No. of testing } \\
\text { samples }\end{array}$ & $\begin{array}{c}\text { Recognition } \\
\text { Accuracy (\%) }\end{array}$ \\
\hline 1 & $20 * 15=300$ & $30 * 15=450$ & 42.88 \\
\hline 2 & $25 * 15=375$ & $25 * 15=375$ & 56.5 \\
\hline 3 & $30 * 15=450$ & $20 * 15=300$ & 62 \\
\hline 4 & $35 * 15=525$ & $15 * 15=225$ & 62.3 \\
\hline 5 & $40 * 15=600$ & $10 * 15=150$ & 62.7 \\
\hline 6 & $45 * 15=675$ & $5 * 15=75$ & $\mathbf{7 2}$ \\
\hline
\end{tabular}

In the next part of the work a total of 9 iterations have been done by varying the various samples of both training and testing sets which were shown in table 2 .

These iterations are done for the validation of results. The average recognition accuracy from table 2 is $71.41 \%$ that is approximately equal to $72 \%$.

Published By:

Blue Eyes Intelligence Engineering \& Sciences Publication

(C) Copyright: All rights reserved 
Table- 2: Validation for recognition accuracy of Hindi samples

\begin{tabular}{|c|c|}
\hline \multicolumn{2}{|c|}{ samples } \\
\hline Iteration & Recognition Accuracy (\%) \\
\hline 2 & 57.3 \\
\hline 3 & 61.4 \\
\hline 4 & 78.66 \\
\hline 5 & 73.33 \\
\hline 6 & 72 \\
\hline 7 & 74.67 \\
\hline 8 & 70.67 \\
\hline 9 & 78.66 \\
\hline
\end{tabular}

The Fig.1 and Fig.2 shows the correctly matched and wrongly matched Hindi script test characters using the proposed algorithm.
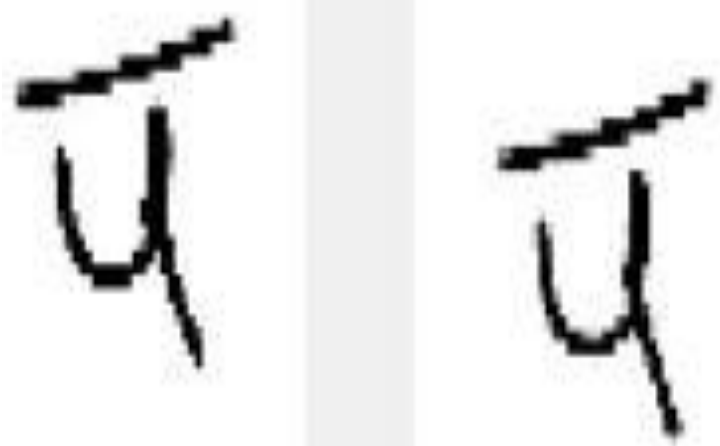

Input image

output image

Fig. 1. Correctly matched Hindi script test images to database images
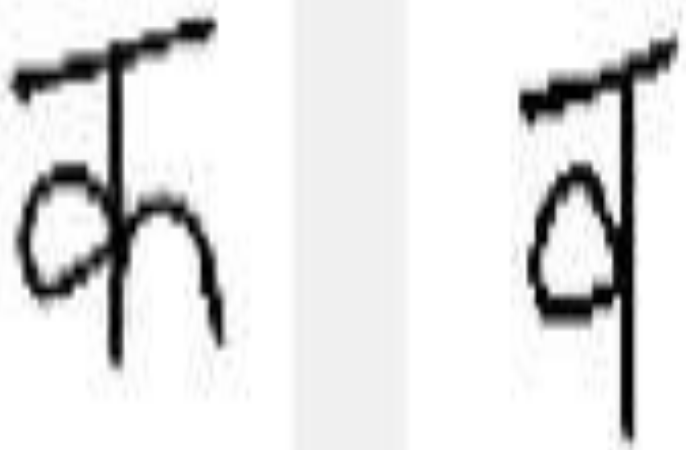

Input image

output image

Fig. 2. Wrongly matched Hindi script test images to database images

The recognition accuracies from table 1 was represented graphically by a bar graph in Fig.3, we can observe that the R.A was highest for the data of 45 number of training samples.

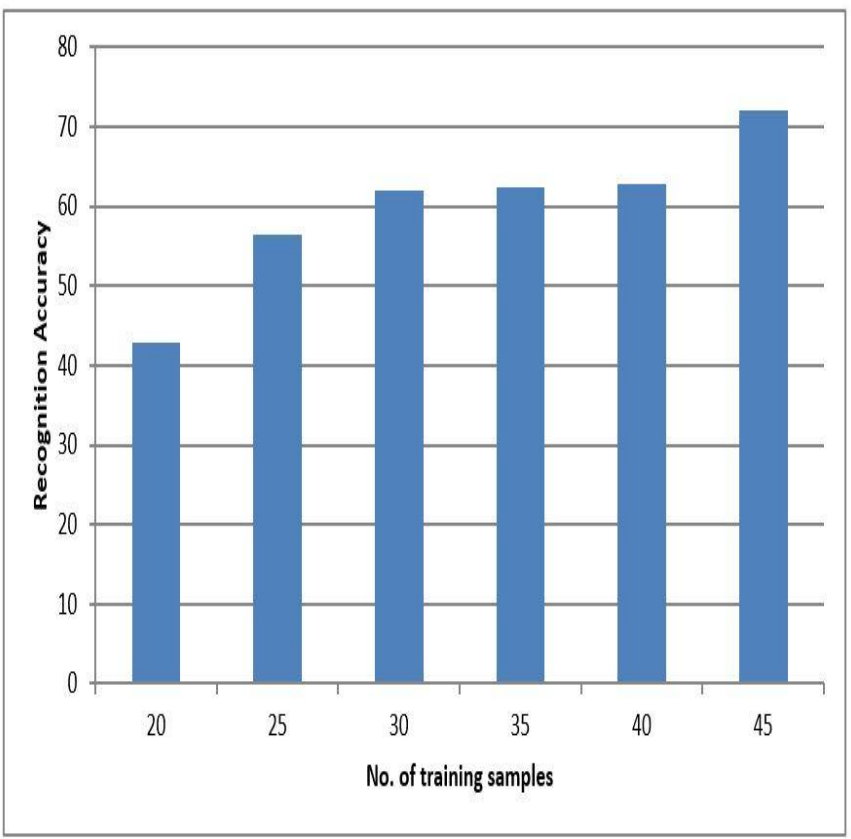

Fig. 3. Graphical representation of experiment 1 for Hindi characters

The table 3 represents the 15 classes of English characters. Each class consists of 50 samples which are divided into training and testing samples as shown in the table. The recognition accuracy was calculated with respect to the number of training samples and number of testing samples. The highest recognition accuracy achieved was 69.33\% for which the number of training samples are 40 and the number of testing samples are 10 .

Table- 3: Experiment 2 for 15 English classes

\begin{tabular}{|c|c|c|c|}
\hline S.no & $\begin{array}{c}\text { No of training } \\
\text { samples }\end{array}$ & $\begin{array}{c}\text { No of testing } \\
\text { samples }\end{array}$ & $\begin{array}{c}\text { Recognition } \\
\text { Accuracy (\%) }\end{array}$ \\
\hline 1 & $20^{*} 15=300$ & $30 * 15=450$ & 61.33 \\
\hline 2 & $25 * 15=375$ & $25 * 15=375$ & 63.2 \\
\hline 3 & $30 * 15=450$ & $25^{*} 15=300$ & 66 \\
\hline 4 & $35 * 15=525$ & $15 * 15=225$ & 65.33 \\
\hline 5 & $40^{*} 15=600$ & $10^{*} 15=150$ & $\mathbf{6 9 . 3 3}$ \\
\hline 6 & $45^{*} 15=675$ & $5 * 15=75$ & 66.666 \\
\hline
\end{tabular}

The recognition accuracy of the 4 iterations have been done and listed in table 4 . These iterations were done to validate the results. The average recognition accuracy of the above iterations was $71.665 \%$. Thus, we can say that R.A of experiment 2 was validated.

Table- 4: Validation for recognition accuracy of English samples

\begin{tabular}{|c|c|}
\hline Iteration & Recognition Accuracy (\%) \\
\hline 1 & 74 \\
\hline 2 & 70.66 \\
\hline 3 & 74 \\
\hline 4 & 68 \\
\hline
\end{tabular}

The Fig. 4 and Fig.5 shows the correctly matched and wrongly matched English script test characters using the proposed algorithm.

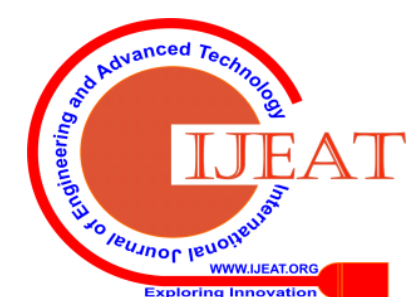




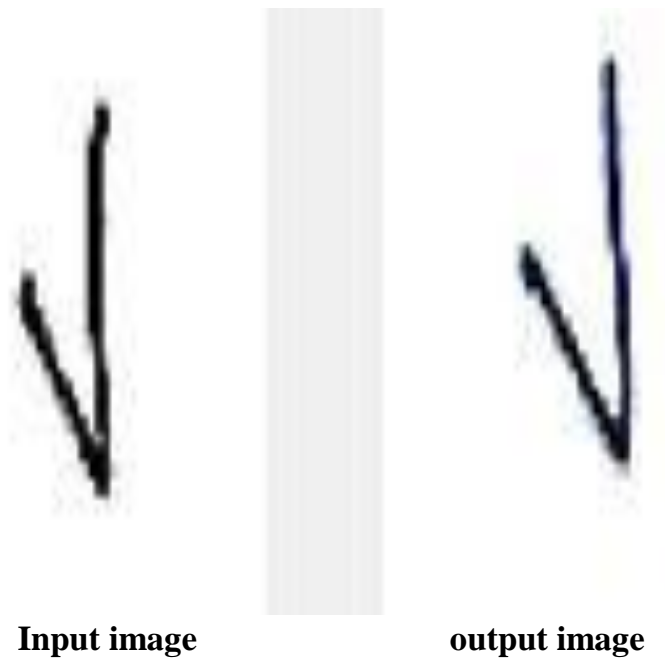

Fig. 4. Correctly matched English script test images to database images
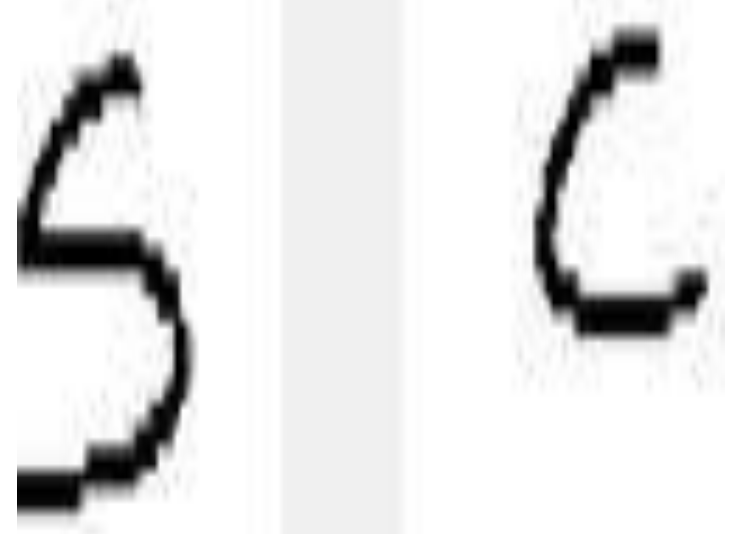

Input image output image

Fig. 5. Wrongly matched English script test images to database images

The recognition accuracies from table 3 was represented graphically by a bar graph in Fig.6, we can observe that the Recognition accuracy was highest for the data of 40 number of training samples.

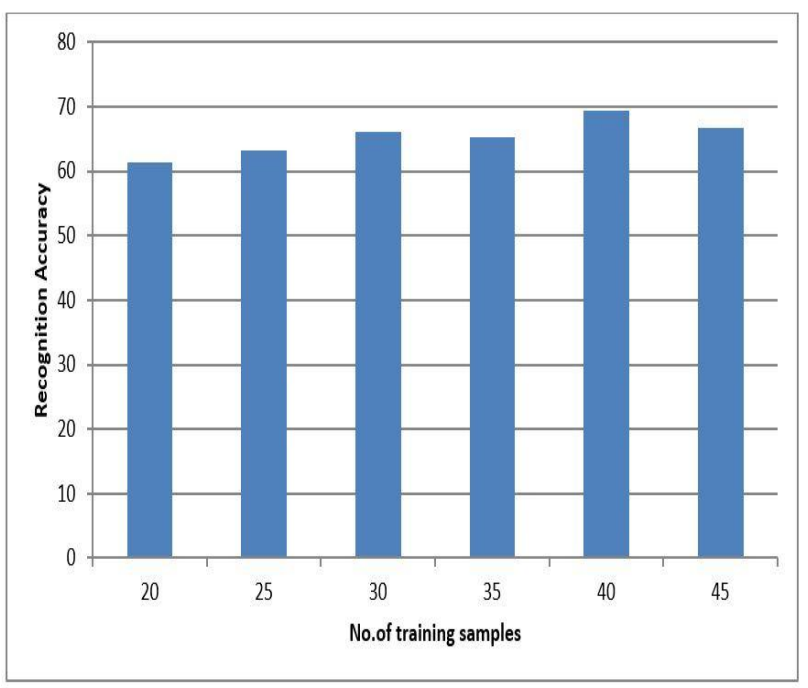

Fig. 6. Graphical representation of experiment 2 for English characters
The table 5 represents the 15 classes of both Hindi and English characters. Each class consists of 50 samples which are divided into training and testing samples as shown in the table 5. The recognition accuracy was calculated with respect to the number of training samples and number of testing samples. The highest recognition accuracy achieved was 70\% for which the number of training samples were 40 and the number of testing samples were 10 .

Table- 5: Experiment 3 for 15 classes of both English \& Hindi classes

\begin{tabular}{|c|c|c|c|}
\hline S.no. & $\begin{array}{c}\text { No. of training } \\
\text { samples }\end{array}$ & $\begin{array}{c}\text { No. of testing } \\
\text { samples }\end{array}$ & $\begin{array}{c}\text { Recognition } \\
\text { Accuracy (\%) }\end{array}$ \\
\hline 1 & $20 * 15=300$ & $30 * 15=450$ & 49 \\
\hline 2 & $25 * 15=375$ & $25 * 15=375$ & 55.733 \\
\hline 3 & $30 * 15=450$ & $20 * 15=300$ & 61.666 \\
\hline 4 & $35 * 15=525$ & $15 * 15=225$ & 61.111 \\
\hline 5 & $40 * 15=600$ & $10 * 15=150$ & $\mathbf{7 0}$ \\
\hline 6 & $45 * 15=675$ & $5 * 15=75$ & 68.666 \\
\hline
\end{tabular}

The recognition accuracy of the 4 iterations have been done and listed in table 6 . These iterations were done to validate that results. The average recognition accuracy of the above iterations was $68.958 \%$. Thus, we can say that R.A of experiment 3 was validated as $68.958 \%$ is approximately equal to $70 \%$.

Table- 6: Validation for recognition accuracy of both Hindi and English samples

\begin{tabular}{|c|c|}
\hline Iteration number & Recognition Accuracy (\%) \\
\hline 1 & 66 \\
\hline 2 & 69.333 \\
\hline 3 & 71 \\
\hline 4 & 69.499 \\
\hline
\end{tabular}

The recognition accuracies from table 5 was represented graphically by a bar graph in Fig.7, we can observe that the Recognition accuracy was the highest for the data of 40 number of training samples.

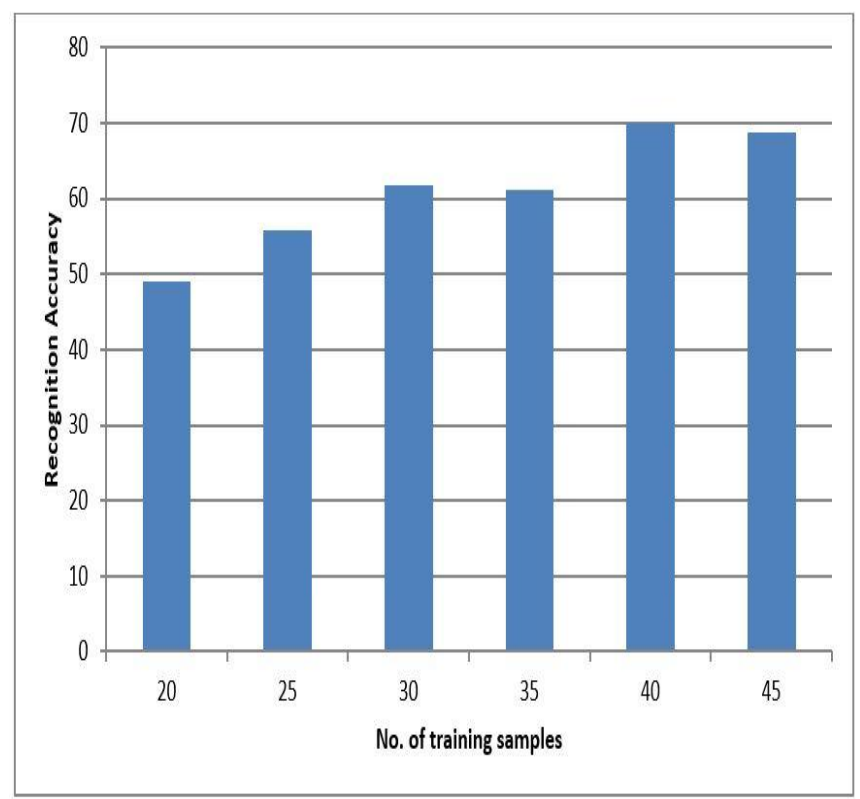

Fig. 7. Graphical representation of experiment 3 for both Hindi and English characters

Published By:

Blue Eyes Intelligence Engineering \& Sciences Publication

(C) Copyright: All rights reserved. 
Table- 7: Comparison between published and proposed method $^{[11]}$

\begin{tabular}{|c|c|c|c|}
\hline s.no & Description & Published method & $\begin{array}{c}\text { Proposed } \\
\text { method }\end{array}$ \\
\hline 1 & $\begin{array}{c}\text { No. of classes } \\
\text { tested }\end{array}$ & 10 & 30 \\
\hline 2 & Script & Devanagari & $\begin{array}{c}\text { English \& } \\
\text { Hindi }\end{array}$ \\
\hline 3 & Feature extraction & wavelet & 2D-FFT \\
\hline 4 & $\begin{array}{l}\text { No. of training } \\
\text { samples }\end{array}$ & 50 & 45 \\
\hline 5 & $\begin{array}{l}\text { No. of testing } \\
\text { samples }\end{array}$ & 15 & 05 \\
\hline 6 & $\begin{array}{c}\text { Recognition } \\
\text { Accuracy }\end{array}$ & $65 \%$ & $70 \%$ \\
\hline 7 & Classifier & Neural networks & $\begin{array}{c}\text { Nearest } \\
\text { Neighborhood } \\
\text { Classifier }\end{array}$ \\
\hline
\end{tabular}

In table 7 the results obtained by the proposed method were compared with the published method. Gaurav Y. Tawde [11] from the published method experimented on Devanagari numerals by extracting the features using wavelet transform. Then the feature extraction was followed by neural network classifier that was used for classification. The average recognition accuracy was reported to be $65 \%$ with 50 training samples/class and 15 testing samples/class. In their work totally 10 samples were tested classes.

In the proposed method the features are extracted using 2D-FFT. The Nearest Neighborhood Classifier was used for classification. The recognition accuracy obtained was 70\% with 45 training samples/class and 5 testing samples/class. The total number of tested classes in the proposed method were 30

\section{CONCLUSIONS}

The work presented in this paper proposes an offline handwritten character recognition system for Hindi and English scripts. The database of both Hindi and English scripts were developed successfully for training and testing datasets. A total number of 3000 samples used which are further normalized to $50 \times 50$ pixels. The classifier that has been employed in this work is Nearest Neighborhood classifier. The recognition accuracy was calculated and then that was validated with different iterations.

The best recognition accuracy obtained using 2D FFT when Hindi characters were considered was $72 \%$ and when English characters were considered was $\mathbf{6 9 . 3 3 \%}$. The best recognition accuracy obtained using 2D FFT for both the Hindi and English characters was $\mathbf{7 0 \%}$.

The further research can be focused on exploring new or hybrid feature extraction methods. The advanced techniques using the neural networks or support vector machine can be used to improve the recognition accuracy of Handwritten Character Recognition.

\section{REFERENCES}

1. V.N. Manjunath Aradhya, G. Hemantha Kumar, and S. Noushath. "Robust unconstrained handwritten digit recognition using radon transform”. In IEEE - ICSCN, pages 626-629, 2007.

2. U. Bhattacharya and B. B. Chaudhuri. "Handwritten numeral databases of indian scripts and multistage recognition of mixed numerals". IEEE transcations on pattern analysis and machine intelligence, 31(3):444 457, Mar, 2009.
3. Panyam Narahari Sastry, T. R. Vijaya Lakshmi, N. V. Koteswara Rao and Rama Krishnan Krishnan. "Analysis of telugu palm leaf characters using multilevel recognition approach". ARPN Journal of Engineering and Applied Sciences. VOL. 10, NO 20, 2015.

4. P. N. Sastry, R. Krishnan. "Isolated Telugu palm leaf character recognition using radon transform, a novel approach," in World Congress on Information and Communication Technologies (WICT), pp. 795-802, 2012.

5. P. N. Sastry, R. Krishnan, B. V. S. Ram, "Telugu character recognition on palm leaves- a three dimensional approach," Technology Spectrum, vol. 2, no. 3, pp. 19-26, 2010.

6. T. R. Vijaya Lakshmi, P. N. Sastry, T. V. Rajinikanth. "Recognition of isolated Telugu Handwritten characters using 2D FFT". In Proceedings of $2^{\text {nd }}$ International Conference on Advanced Computing Methodologies (ICACM), 2-3 Aug. 2013, GRIET, Hyderabad, pp. 372-376

7. P. N. Sastry, T. R. Vijaya Lakshmi, N. V. Koteswara, T. V. Rajinikanth, A. Wahab. "Telugu Handwritten Character Recognition Using Zoning Features," International Conference on IT Convergence and Security (ICITCS), pp.1-4, 2014.

8. Munish Kumar, M. K. Jindal, R. K. Sharma. "k -Nearest Neighbor Based Offline Handwritten Gurmukhi Character Recognition”. In IEEE International Conference on Image Information Processing, 2011.

9. Gayathri P, Sonal Ayyappan. "Off-line Handwritten Character Recognition using Hidden Markov Model”. International Conference on Advances in Computing, Communications and Informatics, 2014.

10. N. Prameela, P. Anjusha, R. Karthik. "Off-line Telugu Handwritten Characters Recognition using optical character recognition". International Conference on Electronics, Communication and Aerospace Technology (ICECA). 2017.

11. Gaurav Y. Tawde, "Optical Character Recognition for Isolated Offline Handwritten Devanagari Numerals Using wavelets", International Journal of Engineering Research and Applications, 2248-9622, Vol. 4, Issue 2 (Version 1), pp.605-611, February 2014.

12. Pritpal Singh et al. "Handwritten Gurmukhi Character Recognition Using Wavelet transforms", International Journal of Electronics, Communication \& Instrumentation Engineering Research and Development (IJECIERD) ISSN 2249-684X, Vol.2, Issue 3, Sep. 2012, 27-37.

\section{AUTHORS PROFILE}

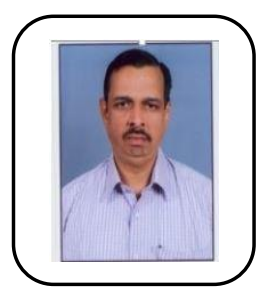

Dr. P. Narahari Sastry is working in CBIT since 2003, and is involved in Teaching and Research. He has recognized as Supervisor for JNTU Hyderabad, Osmania University Hyderabad and K.L. University Vijayawada. He has guided three (03) PhD students and also guiding eight (8) Research scholars from Osmania University Hyderabad presently. He has published 6 SCI Journals in the last 5 years and 2 of them are published in the reputed journals which are "Electrical and Computer Engineering" and IEEE Communication letters. Also he has applied and published his work in Indian Journal of patents, Chennai, India. He was the PI for an AICTE project titled "Design and Development of Palm Leaf Character Recognition System" granted under RPS scheme, which was successfully completed during the period 2013-16.

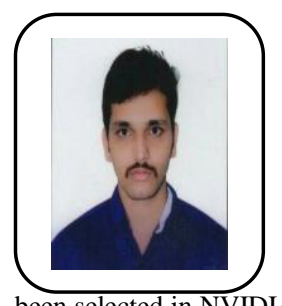

Syed Sameer is currently pursuing Post-Graduation (PG) i.e. M.E final year in Electronics and Communication Engineering (ECE) department at Chaitanya Bharathi Institute of Technology (CBIT-A), Hyderabad, India. He is interested in Image Processing and Research on Languages. He has qualified for Council of Scientific and Industrial Research (CSIR). He has been selected in NVIDIA as a Process Executive. 


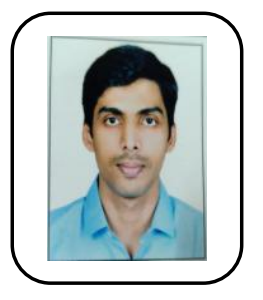

Mohammed Sameer Syed is currently pursuing Post-Graduation (PG) i.e. M.E final year in the Electronics and Communication Engineering (ECE) department at Chaitanya Bharathi Institute of Technology (CBIT-A), Hyderabad, India. He has keen interest in the field of image processing. He worked on projects related to communication, antennas, IoT, neural networks and pattern recognition. He was an intern in BSNL, Tirupati. He is also doing a research project in the field of machine learning with python programming software.

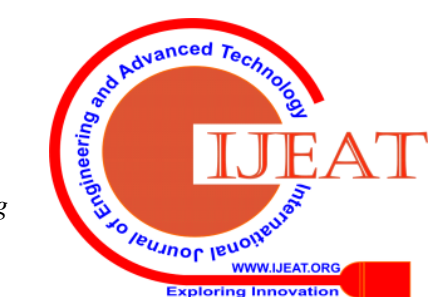

